« Numerical study of effective heat conductivities of foams by coupled conduction and radiation $»$

by

G. L. VIGNOLES \& A. ORTONA,

published in

Int. J. Therm. Sci. (2016), vol. 109, pp. 270-278.

DOI : $\underline{10.1016 / j . i j t h e r m a l s c i .2016 .06 .013 ~}$

http://www.sciencedirect.com/science/article/pii/S1290072916302022 


\title{
Numerical study of effective heat conductivities of foams by coupled conduction and radiation
}

\author{
Gerard L. Vignoles ${ }^{\mathrm{a}}$, Alberto Ortona ${ }^{\mathrm{b}}$ \\ ${ }^{a}$ University of Bordeaux, \\ Laboratoire des Composites ThermoStructuraux (LCTS) \\ UMR 5801: CNRS-Herakles(Safran)-CEA-UBx \\ 3, Allée de La Boétie, 33600 Pessac, France \\ Tel: (+33) 556843305 \\ ${ }^{b}$ iCIMSI, SUPSI, Manno, Switzerland
}

\begin{abstract}
Effective thermal conductivities (ETC) under vacuum were computed numerically on 3D blocks of open-cell foams either obtained by X-ray tomography or generated by Computer-Aided Design (CAD) with ideal geometries.For the first time a Monte-Carlo/Random Walk code accounting for the coupling of conduction in the solid phase and of radiation in the pore space has been used. The whole range of conduction/radiation ratio, parameterized by a Nusselt number, has been scanned; a law relating the ETC to this parameter has been obtained. In all cases the conductive and radiative contributions are additive. The slope of the radiative contribution to the ETC is found to display a distinct behavior, depending on whether radiation or conduction dominates. The low-temperature regime has an emissivity-dependent ETC slope, while the high-temperature regime does not. The critical ratio between both regimes is related to the ratio between cell and strut diameters. In all cases, it is found that the ETC anisotropy decreases with temperature. Closing some windows enhances conduction parallel to the closing walls and reduces radiation perpendicular to them. This effect is shown to influence the
\end{abstract}

Email address: vinhola@lcts.u-bordeaux1.fr (Gerard L. Vignoles) 
ETC eigendirections in actual media.

Keywords: Coupled heat transfer ; Radiative heat transfer ; Effective Thermal Conductivity; Virtual material ; Foams

\section{Highlights}

- Effective conductivity computations by new, original scheme

- 3D X-ray tomography blocks compared to ideal virtual materials

- Radiative conductivity excess has two limiting regimes (low $T$ / high $T$ )

- Material anisotropy decreases with temperature

- Effect of closing some cell windows is described

\section{Introduction}

Open-cell foams are currently used in many engineering applications [1] related to heat transfer. Polymer foams are used as insulators [2-4], metal foams as compact heat exchangers [5], ceramic foams as porous burners [6, 7] or solar collectors [8-10], carbon foams as heat exchangers [11] or electrodes [12], especially for fuel cells [13]. Carbon [14] or metal [15] foams are used as a conductive network and as mechanical reinforcement for thermal energy storage materials, in combination with phase change materials (PCM).

Heat transfer properties are in focus for these applications, especially the effective thermal conductivity (ETC). It depends on the foam structure and on the physics of heat transfer : conduction in the solid, conduction and convection in the medium filling the void spaces, and radiation in the transparent or semitransparent phases [16]. Evaluation of these properties has been the subject of abundant literature, both on the experimental and modeling sides. We refer the 
reader to recent reviews on the subject, for instance, a very broad review of analytical and empirical models for aluminum foams $[17,18]$.

Models for the ETC of open-cell foams are either analytical (based on simple geometrical assumptions), empirical (i.e. fitted to experimental trends), or based on numerical computations.

Empirical models, based on the Rosseland approximation [19] and featuring an effective extinction coefficient, have been satisfactorily fitted to experimental data [20,21]. The contribution of radiation to the thermal properties has thus been evaluated for polymer [22-24] and carbon [25] foams. Though very useful, these empirical models contain adjustable parameters and are not able to give precise insight into the physics of heat transfer; structure-based or image-based modeling has therefore been undertaken in parallel with these correlations in order to extract more physical information.

By far, models restricted to pure conduction are the most numerous ones. Following very early work [26], analytical models are generally based on the simplification of a given structure into a network of thermal resistances. The foams are generally considered as saturated by a fluid; accordingly, the models depend on solid and fluid intrinsic conductivities, plus some geometrical parameters that can be as simple as just porosity, or more elaborate [18]. Many geometries have been analyzed: arrangements of cubes with or without spherical holes [27], hexagonal cells [28], fiber assemblies [29, 30], symmetrical interconnected network of fibers and cavities[31], etc .... Boomsma \& Poulikakos[32] reported a first model on tetrakaidecahedra; corrections and extensions were suggested by Dai \& Nawaz $[33,34]$; a similar approach has been proposed, extended to different strut section geometries [35].

Numerical computations on large resolution domains becoming increasingly easy, estimations are produced by the resolution of the heat equation. Kumar and 
Topin [36] chained Computer-Aided Design (CAD) and Finite Elements (FE) solution with commercial software to produce 2000 data points. Recent CAD+FE computations on Laguerre-Voronoï cells [37] match satisfactorily experimental results. Direct computations on X-ray Computed Micro-Tomography (CMT) images have been also carried out. Bodla \& Murthy [38] extracted an equivalent resistance network from a scan and solved quasi-analytically the associated transfer problem. FE computations have also been conducted [39] ; agreement with experimental determinations has been obtained [40].

On the other hand, the modeling of purely radiative effects has been investigated separately during many years.

The computation of effective radiative properties (extinction, scattering coefficients and phase function) has been performed from structural parameters estimated by 3D image analysis [41]. Numerical estimations were also directly obtained from CAD or CMT images [42-44], using the Radiative Distribution Function Identification (RDFI) method [45]. Monte-Carlo methods [46] are generally used for this type of computation. For instance, a recent work [47] reviews the existing correlations between the radiative conductivity, the porosity and the surface area per unit volume, and proposes a new one, thanks to new computations. These methods focused on radiative heat transfer are almost purely geometric methods; their difference in essence with "deterministic" methods makes them extremely difficult to couple intimately with classical heat equation solvers.

Modeling efforts on coupled conductive-radiative transfer have principally addressed the joint solution of the conductive heat equation and of the radiative transfer equation (RTE). The coupling is obtained by the insertion of the radiative power as a source term in the heat equation on the one hand and by the reinsertion of the obtained temperatures in the RTE [48, 49]. Most of these studies have concerned semi-transparent media. A generalization of the RTE approach (GRTE) 
has been derived on opaque/transparent (OT) media [50, 51]. Leroy and Taine [52] have also dealt with OT media including conduction and even convection in the fluid, by coupling the GRTE with the Volume Averaging Method; interesting results have been produced on an ideal simple geometry.

Very recently, Vignoles [53] has proposed a new hybrid Monte-Carlo/Random Walk solver for the computation of effective heat conductivities, accounting for a full, intimate coupling between radiation and conduction. Validations with respect to analytical cases have been obtained.

The purpose of the present study is to investigate numerically, using this new method, the ETC in open-cell foams under mixed conduction/radiation heat transfer. Either 3D images from CAD (ideal foams) or from CMT (actual foams) will be studied, in view of obtaining structure-properties relationships.

The first section will describe the investigated numerical materials and method. In a second part, the nature of the ETC dependence on radiation/conduction ratio will be presented and discussed. Finally, the results obtained on all foams will be given and attempts to correlate them to the foams structural parameters will be made.

\section{Numerical materials and method}

\subsection{Numerical materials}

All computations have been carried out using 3D images considered as numerical resolution domains. The images are considered as structured grids of $N_{x} \times N_{y} \times N_{z}$ voxels. Two types of images were used. First, actual foams were scanned by X-ray CMT. The samples $A \_011$ and $A \_015$ are $S i-S i C$ foams that had been prepared by slurry impregnation, pyrolysis and reactive silicon infiltration on reticulated polyurethane foam templates [54]. Sample $B_{-} 011$ is a CMTscanned polymer foam that has been numerically densified following a "numerical 
replica" technique [55]. It has a much more regular window/strut structure than the $A_{-} x x x$ samples. Table 1 summarizes their main geometrical characteristics. Since these foams have respectively $11 \%$ and $15 \%$ solid volume fraction, it has

\begin{tabular}{cl|c|c|c}
\hline Sample & & B_011 & A_011 & A_015 \\
\hline \hline Voxel size & $(\mathrm{mm} / \mathrm{vox})$ & 0.015 & \multicolumn{2}{|c}{0.032} \\
\hline \multirow{2}{*}{ Dimensions } & & 500 & 256 & 244 \\
& & 500 & 256 & 244 \\
Volume & $\left(\mathrm{mm}^{3}\right)$ & 421.9 & 339.3 & 359.0 \\
\hline Relative density & - & 0.1153 & 0.1103 & 0.1529 \\
\hline Surface per unit volume & $\left(\mathrm{vox}^{-1}\right)$ & 0.0274 & 0.0386 & 0.0410 \\
& $\left(\mathrm{~mm}^{-1}\right)$ & 1.8267 & 1.2067 & 1.2806 \\
\hline Approx. strut diam. & $(\mathrm{pix})$ & 16.233 & 11.940 & 13.560 \\
& $(\mathrm{~mm})$ & 0.2435 & 0.434 & 0.382 \\
\hline Approx. cell diam. & $(\mathrm{pix})$ & 129.98 & 91.65 & 84.048 \\
& $(\mathrm{~mm})$ & 1.950 & 2.933 & 2.690 \\
\hline
\end{tabular}

Table 1: Geometrical characteristics of CMT images of actual $S i-S i C$ foams

been decided to compare them to the most classical foam models, i.e. tetrakaidecahedra with cylindrical strut sections and corresponding relative densities. In addition to isotropic model foams, slightly elongated models have been produced, with a 1:1:1.4 or 5:5:7 dimension ratio, mimicking the actual $40 \%$ stretching ratio observed on the samples along the rise direction. These actual and ideal foams are rendered in Figure 1.

Finally, two ideal foams has been considered, with only $3 \%$ solid volume fraction, either with all open windows, as in a classical model, or with some closed windows, as illustrated in Figure 2. The idea was to investigate the effect of oc- 


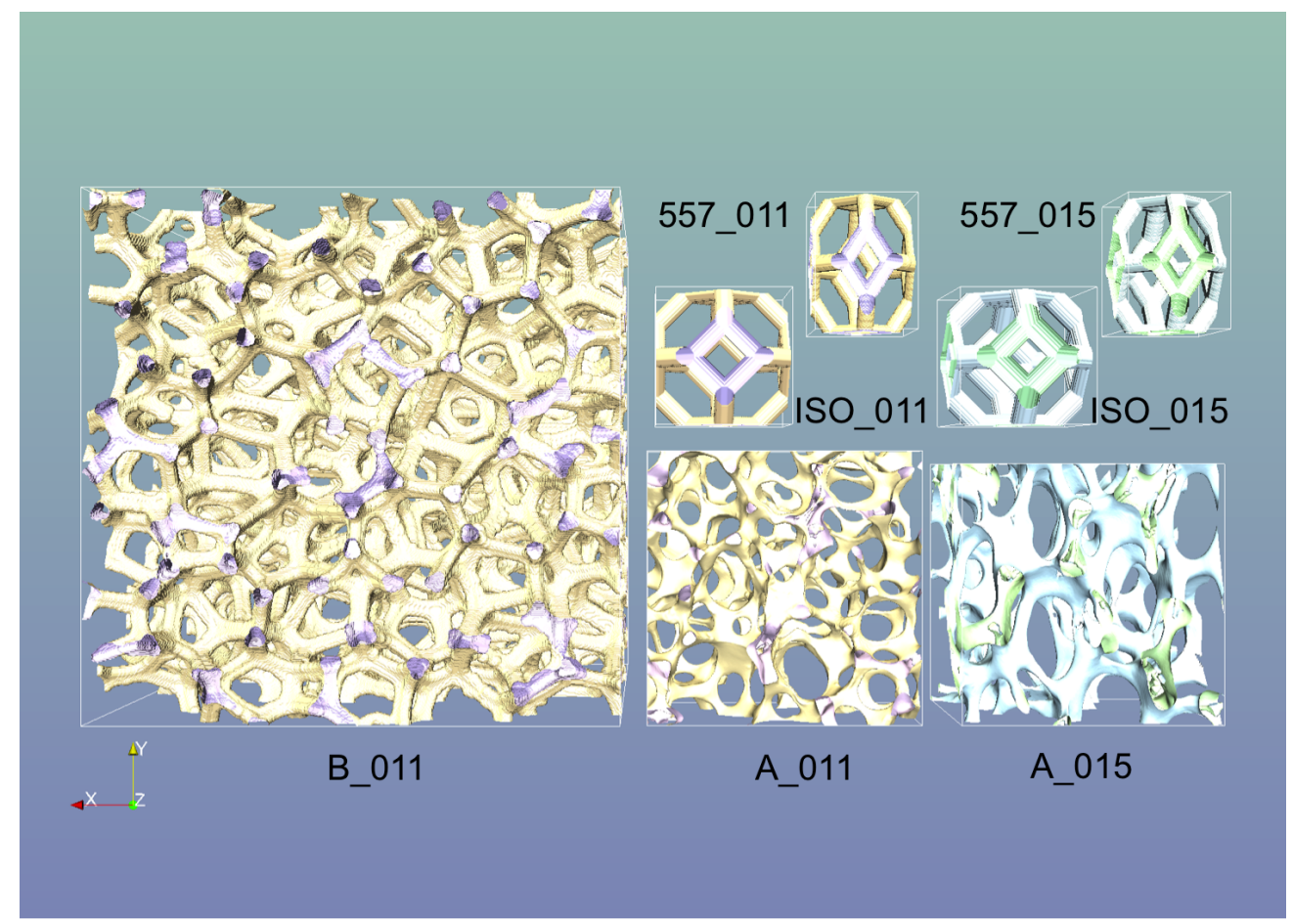

Figure 1: Foams with 11\% solid (yellow, left) and 15\% solid volume fraction (blue,right). Model foams (tetrakaidecahedra) are either isotropic (prefix "ISO") or elongated (prefix "557"). The largest foam is 500 voxels wide; all foams are displayed with the same voxel size. 
culting some windows on heat transfer. Indeed, this phenomenon is unavoidable when preparing foams by the replica technique, in which a polymeric template is dipped into a viscous slurry. When the cohesive forces in the slurry are strong enough, they do not allow to break the film that forms during slurry draining and before its drying. Some closed windows can be seen on sample $A_{-} 015$ (Figure 1 bottom right).

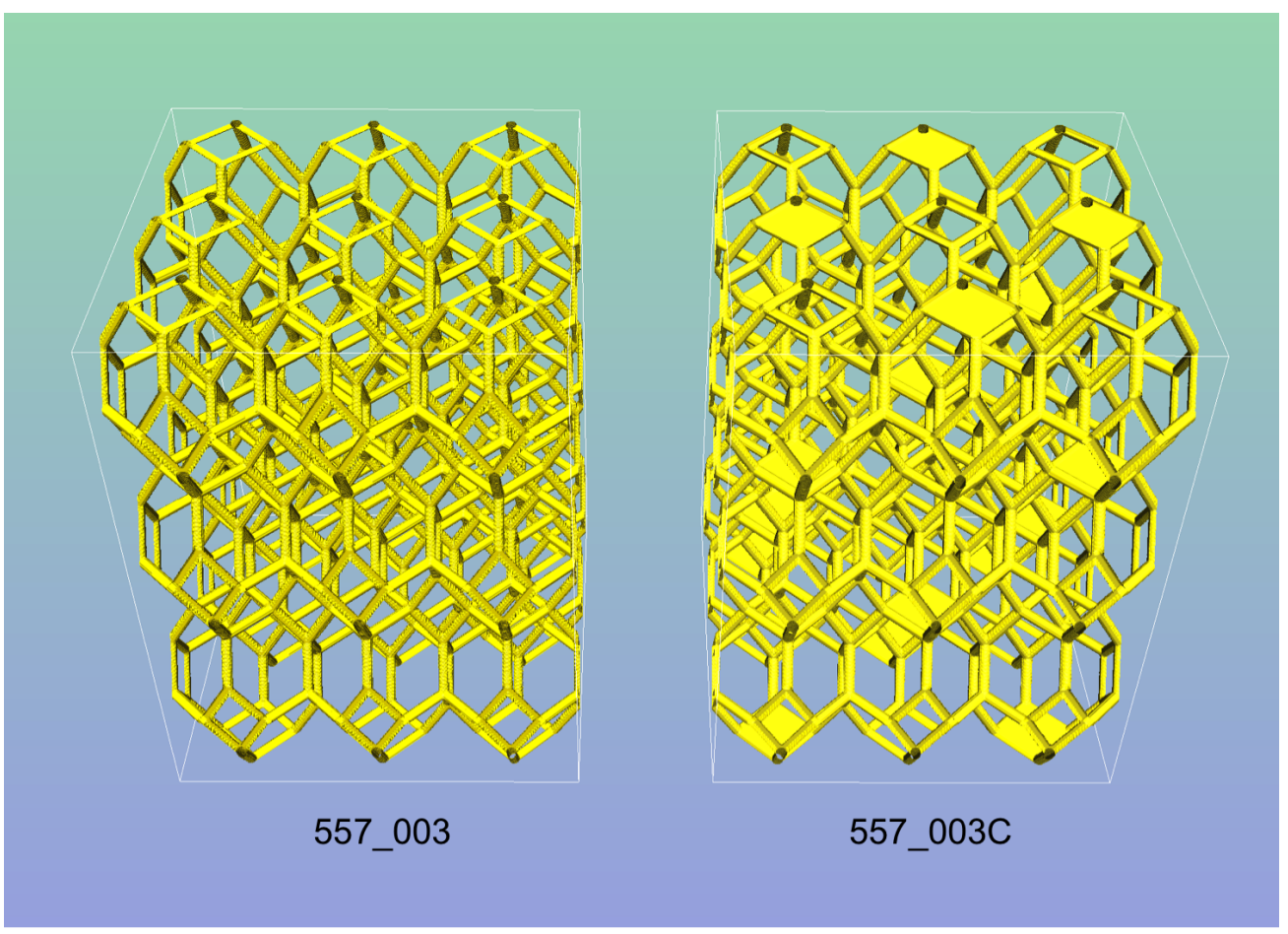

Figure 2: Model foams (tetrakaidecahedra) with with 3\% relative density and open cells (left) or some closed cells (right).

The geometrical characteristics of all ideal foams are summarized in Table 2.

\subsection{Numerical method}

The images were easily binarized and the solid/void interface was discretized according to the Simplified Marching Cube scheme [56]. This technique allowed 


\begin{tabular}{|c|c|c|c|c|c|c|c|}
\hline Sample & & 557_003 & 557_003C & $I S O \_011$ & 557_011 & $I S O \_015$ & 557_015 \\
\hline \multirow{3}{*}{ Dimensions } & \multirow{3}{*}{ (vox) } & 375 & 375 & 128 & 191 & 256 & 191 \\
\hline & & 375 & 375 & 128 & 191 & 256 & 191 \\
\hline & & 500 & 500 & 128 & 256 & 256 & 256 \\
\hline Rel. density & - & 0.0270 & 0.0301 & 0.1010 & 0.1107 & 0.1496 & 0.1458 \\
\hline Surface per unit vol. & $\left(\operatorname{vox}^{-1}\right)$ & 0.0125 & 0.0131 & 0.0234 & 0.0298 & 0.0273 & 0.0328 \\
\hline Approx. strut diam. & (pix) & 8.600 & 9.210 & 17.236 & 14.830 & 21.923 & 17.791 \\
\hline Approx. cell diam. & (pix) & 310.296 & 297.069 & 153.42 & 119.19 & 124.624 & 104.247 \\
\hline
\end{tabular}

Table 2: Geometrical characteristics of computer-generated foams

computing the relative density $\Phi=\rho / \rho_{s}$, the internal surface area $S_{v}$, and rough estimates of the strut diameter $d_{s t}=4 \Phi / S_{v}$ and cell diameter $d_{c}=4(1-\Phi) / S_{v}$.

Once the binarized image has been transformed into a biphasic resolution domain, the effective diffusivity has been computed by the Monte-Carlo Hybrid Random Walk scheme described in [53] and using Einstein's formula. The method has been applied with 24000 random walkers, walking through the images during a dimensionless time $t * a_{s} L^{-2}=2.5$ (where $a_{s}$ is the solid-phase diffusivity and $L$ the image edge size) using a "periodic+random tangential shift" boundary condition, so that the whole space is paved by repetitions of the image, and that the principal axes of the effective transfer are respected. The conductivity is then obtained through multiplication by the heat capacity. There has been no problem in running computations in $500^{3}$ pix $^{3}$ images, with typical resolution times of 10 minutes on a single CPU. To get a typical accuracy of $1 \%$, which suffices for the present purpose, the computational time was chosen long enough - for instance, figure 3 shows that $t>L^{2} / a_{s}$ is sufficient - and the number of walkers was varied until finding a convenient value. 


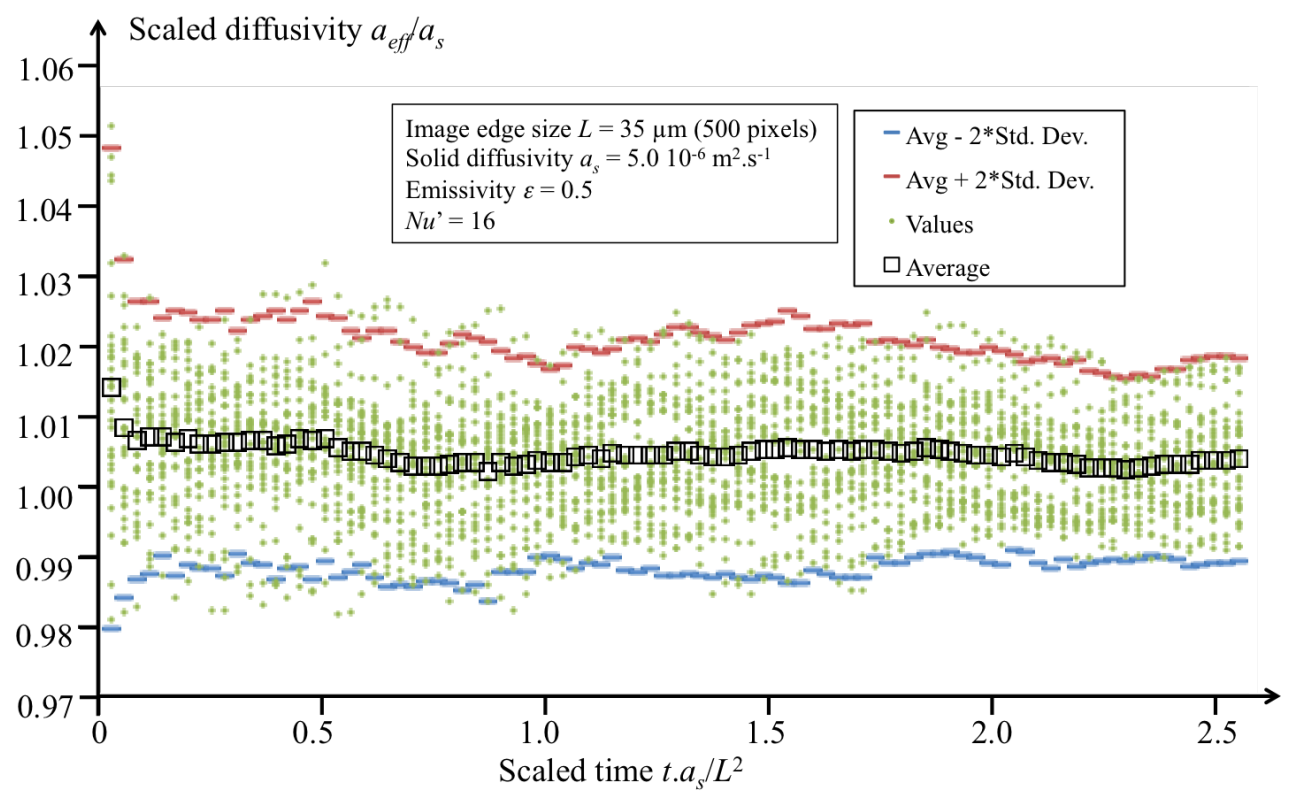

Figure 3: Evolution of the Monte-Carlo results with computational time. The image was $B_{-} 011$ and conditions are given in the insert. Average (empty squares) over 40 runs (dots) and Bollinger band (horizontal marks: \pm 2 standard deviations). 


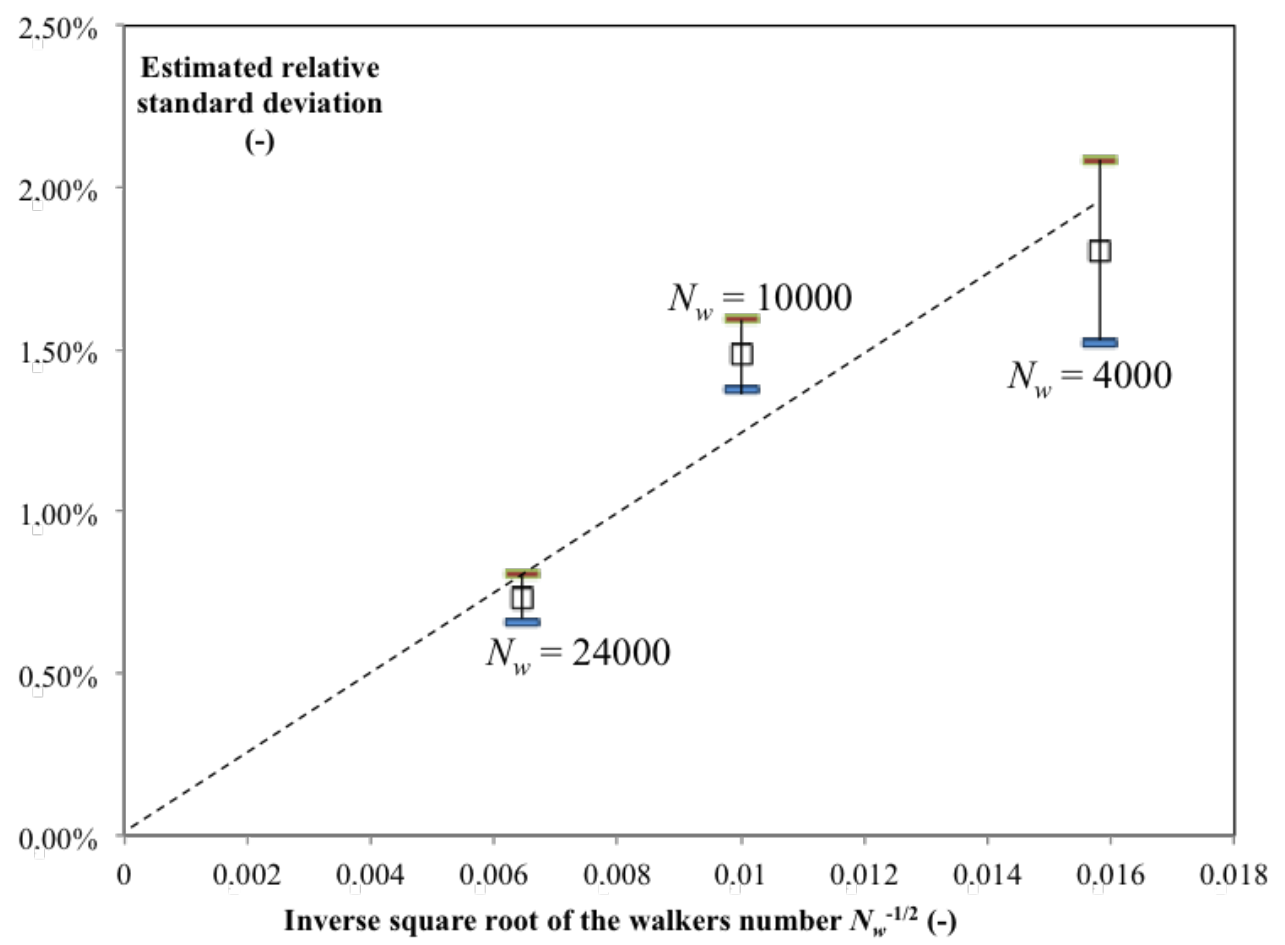

Figure 4: Evolution of the standard deviation of the Monte-Carlo results with the inverse square root of the number of walkers. The image was $B_{-} 011$ and conditions are given in the insert. Standard deviations were averaged over $t . a_{s} / L^{2} \in[1.5 ; 2.5]$ (empty squares) computed over 40 runs (see Figure 3 ) and confidence intervals (between horizontal marks) are \pm 2 standard deviations. 
The results are effective tensors of diffusivity - or of conductivity, obtained by multiplying by a constant heat capacity per unit volume -, the principal axes of which have been determined. The intrinsic directions of fastest, intermediate and slowest heat transfer (eigenvalues and eigenvectors) are thus obtained straightforwardly.

\section{Results and discussion}

\subsection{Influence of temperature on the excess radiative conductivity}

In all results, the conductivities will be scaled with respect to the solid-phase intrinsic conductivity $k_{s}$. The scaled thermal conductivity $\tilde{k}_{e f f}=k_{e f f} / k_{s}$ has been plotted $v s$. the "radiation/conduction ratio", which is an equivalent Nusselt number $[53,57]$ :

$$
N u^{\prime}=\frac{4 \sigma T^{3}}{k_{s}} \cdot \frac{\Pi}{S_{v}}
$$

where $\Pi=1-\Phi$ is the porosity and $S_{v}$ the internal surface area per unit volume, the ratio between both being a length scale close to the cell diameter (actually, 1/4 of it according to our previous estimation ). Note that this $N u^{\prime}$ for a given foam varies only with temperature.

Figs. 5 and 6 illustrate the typical results obtained as the radiation/conduction ratio varies. Figure 5 is a log-log plot of the effective conductivity in the fastest direction. Clearly, a purely conductive limit appears at $N u^{\prime}=0$; then the effective conductivity increases, almost linearly with $N u^{\prime}$ (the slope of the log-log curve is very close to unity). Figure 6 is a plot of the fastest/lowest and mid/lowest eigenvalue ratios, which are measures of the degree of anisotropy of heat transfer. It shows that $(i)$ there is one direction of faster heat transfer (probably the extrusion direction of the foam), the other two being equivalent, and (ii) the anisotropy of this material decreases when the radiative contribution increases, a very general fact. 


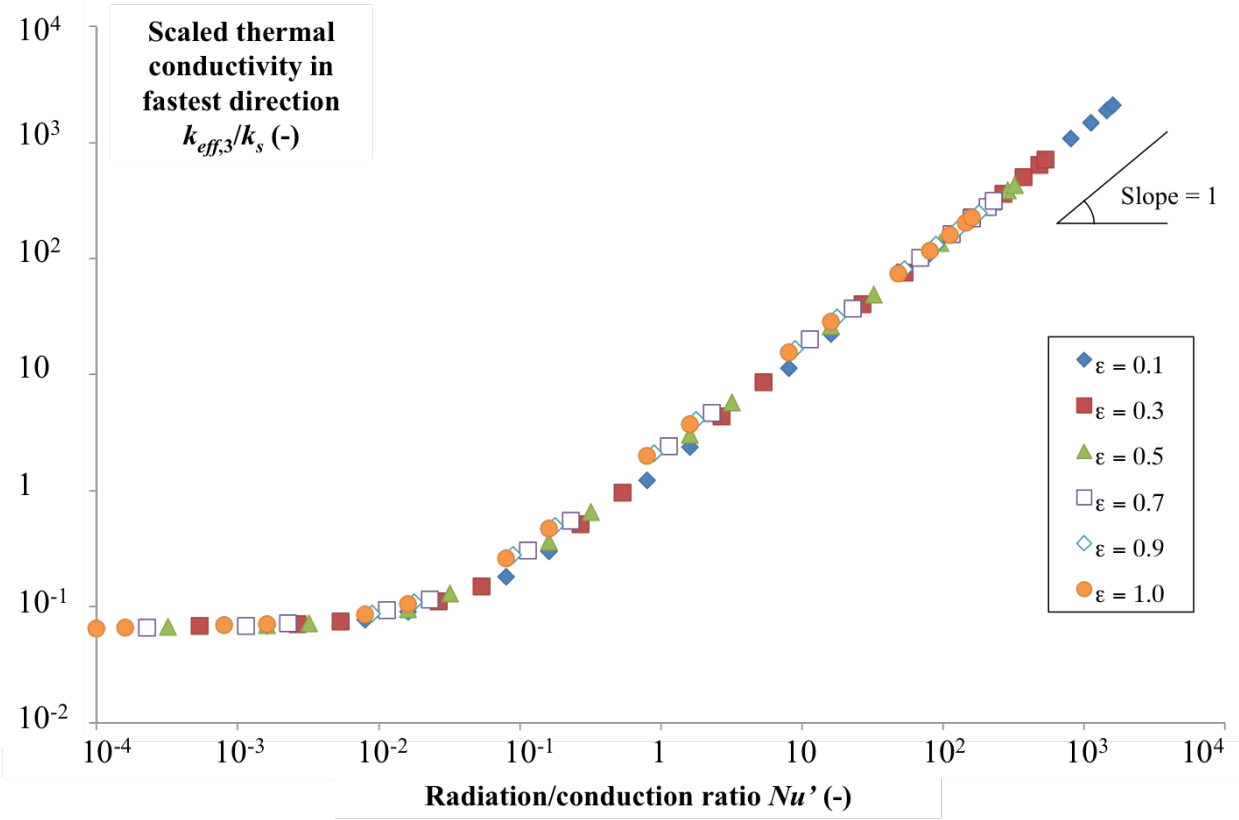

Figure 5: Scaled conductivity $k_{e f f, 3} / k_{s} v s$. radiation/conduction ratio $N u^{\prime}$ for sample $B_{-} 011$ in fastest direction of heat transfer. Every data point is a single computation performed with different values $N u^{\prime}$ and of the emissivity $\varepsilon$, as indicated in the legend. 


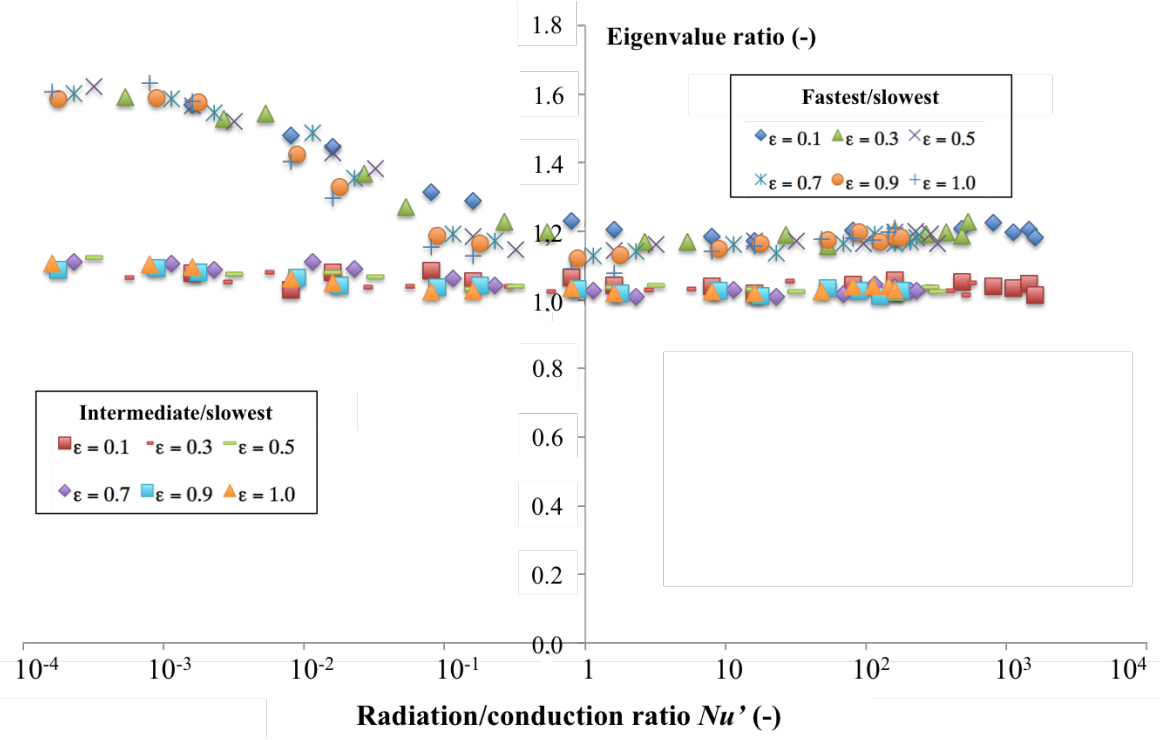

Figure 6: Eigenvalue ratios vs. radiation/conduction ratio $N u^{\prime}$ for sample $B_{-} 011$. Every pair of data points is a single computation performed with different values $N u^{\prime}$ and of the emissivity $\varepsilon$, as indicated in the legends for the fastest/slowest and intermediate/slowest ratios, respectively depicted by the upper and lower sets of data points. 
To interpret Figure 5, we can use the classical Rosseland model:

$$
\tilde{k}_{e f f}=\tilde{k}_{c o n d}+N u^{\prime} \cdot k^{+}
$$

Up to a multiplicative constant, the factor $k^{+}$could be understood as the inverse of an effective dimensionless optical thickness $\beta_{\text {eff }} . L$, where the characteristic length $L$ is $4 \Pi / 3 S_{v}$. (Note that some authors [20] also call $\beta_{e f f} . L$ an effective specific extinction coefficient $E$ ). Relation (2) would fit approximately the data points, especially at very high and very low $N u^{\prime}$ values. However, in the intermediate region there is a discrepancy, that seems to depend on the emissivity. This can be translated by the fact that $k^{+}$is not a single constant, nor a pure function of the emissivity $\varepsilon$ as given in classical literature [58], but instead that it is also a function of $N u^{\prime}$. Withdrawing the purely conductive contribution $\tilde{k}_{c o n d}$ and dividing by $N u^{\prime}$ gives curves like Figure 7, where the obtained $k^{+}$is plotted against $N u^{\prime}$.

At very low $N u^{\prime}, k^{+}$is difficult to estimate with precision, because of the extremely low probability to radiate heat. Indeed, in that case, the radiative contribution is very small with respect to the conductive contribution. For $N u^{\prime}>10^{-2}$ (see figure 5 , it becomes appreciable. In the $\left[10^{-2} ; 10^{4}\right]$ interval, a critical $N u^{*}$ appears, delimiting the two physical regimes:

- at high $N u^{\prime}$ (purely radiative limit, $N u^{\prime}>>N u^{*}$ ), a constant value appears, that does not depend on the emissivity.

- at intermediate or low $N u^{\prime}\left(N u^{\prime}<<N u^{*}\right)$, there is a low-radiation limit, that is larger than the high-radiation limit and varies linearly with the emissivity:

$$
k_{\text {low }}^{+}=k_{\infty}^{+}+\varepsilon \Delta k^{+}
$$

where $\varepsilon \Delta k^{+}$is the additional radiative conductivity slope. 


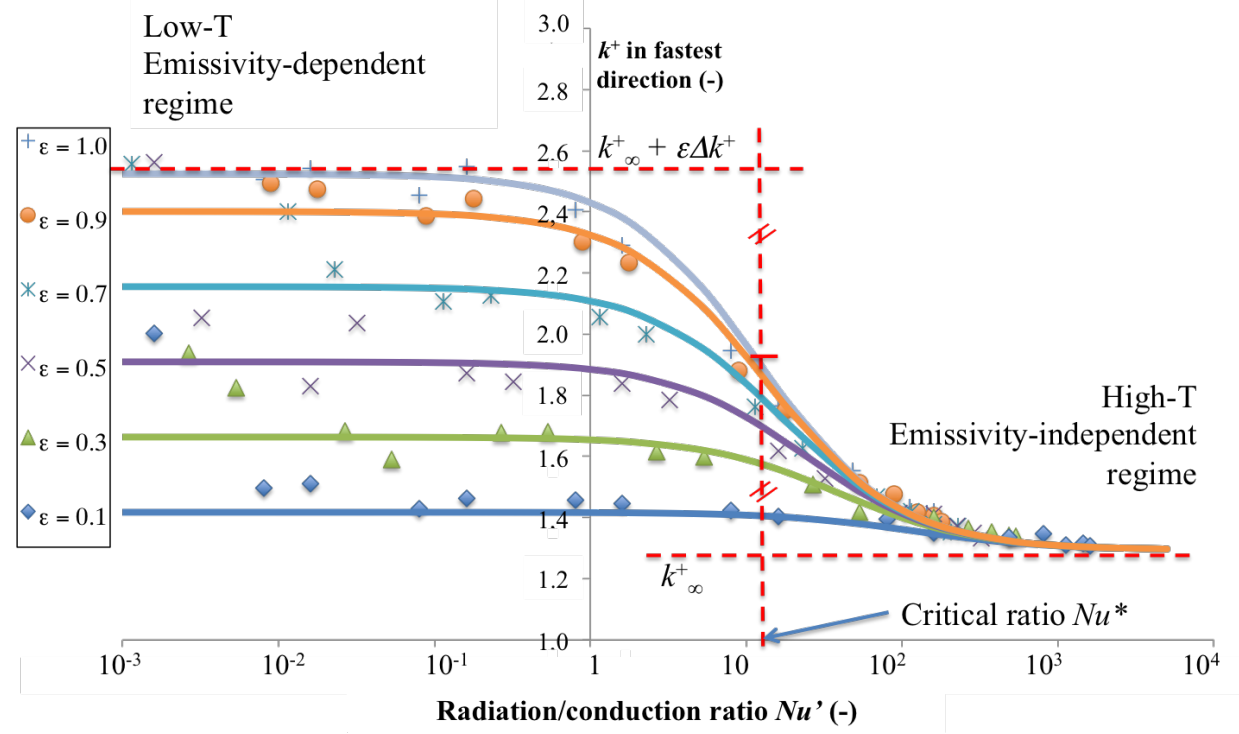

Figure 7: Scaled radiative conductivity slope $k^{+}$(see eq. 2) vs. radiation/conduction ratio $N u^{\prime}$ for sample $B_{-} 011$ and different values of emissivity. The continuous lines are obtained by a least squares fitting of eq.(4) to all data points. 
The critical value $N u^{*}$ is obtained graphically as the abscissa of the vertical segment bounded by the two horizontal asymptotes that intersect the curve in its middle. A physical explanation of this transition could be the following one: when $N u^{\prime}$ is large with respect to $N u^{*}$, one can consider that most of the temperature drop through a given slab of a medium traversed by the heat flux is "trapped" inside the solid. Indeed, surface elements in direct line of sight through the void equilibrate well their temperatures, while their temperature difference can be appreciable if they are opposite faces of some part of the (more resistive) solid here, the ligaments.On the other hand, if $N u^{\prime}$ is comparable or less than $N u^{*}$, the radiating cavities participate appreciably to the overall temperature drop. It is therefore inferred that $N u^{*}$ should be defined by $\frac{d_{c}}{d_{s t}} \approx \frac{\Phi}{1-\Phi}$, and actually it is exactly the case, as illustrated in figure 8 . In practice, we should expect to see this transition in extremely porous media, in which the $\mathrm{Nu}^{*}$ ratio is high; otherwise, the transition would occur when the radiative contribution to heat transfer isn't yet dominant enough.

Using a least squares minimization over the whole data set ,the computed values have been fitted satisfactorily by the following expression:

$$
k_{e f f} / k_{s}=k_{\text {cond }} / k_{s}+N u^{\prime} \cdot k^{+}=k_{\text {cond }} / k_{s}+N u^{\prime} k_{\infty}^{+}+\frac{\Delta k^{+}}{1 / N u^{*}+1 /\left(\varepsilon N u^{\prime}\right)}
$$

This looks like a "series + parallel" model, illustrated at Figure 9. Three branches are in parallel: a first one associated to conduction, a second one associated to radiation when it dominates conduction, and a third one that contains two resistances in series, giving an additional radiative conductivity $\Delta k^{+}$in the case of dominating conduction. This third branch is inactive at high temperatures. It expresses an intimate coupling effect between conduction and radiation due to the complex pore geometry, and had never been reported before by lack of adequate computational methods for this type of local-scale coupling.

So, the conducto-radiative heat conductivity in these opaque/transparent porous 


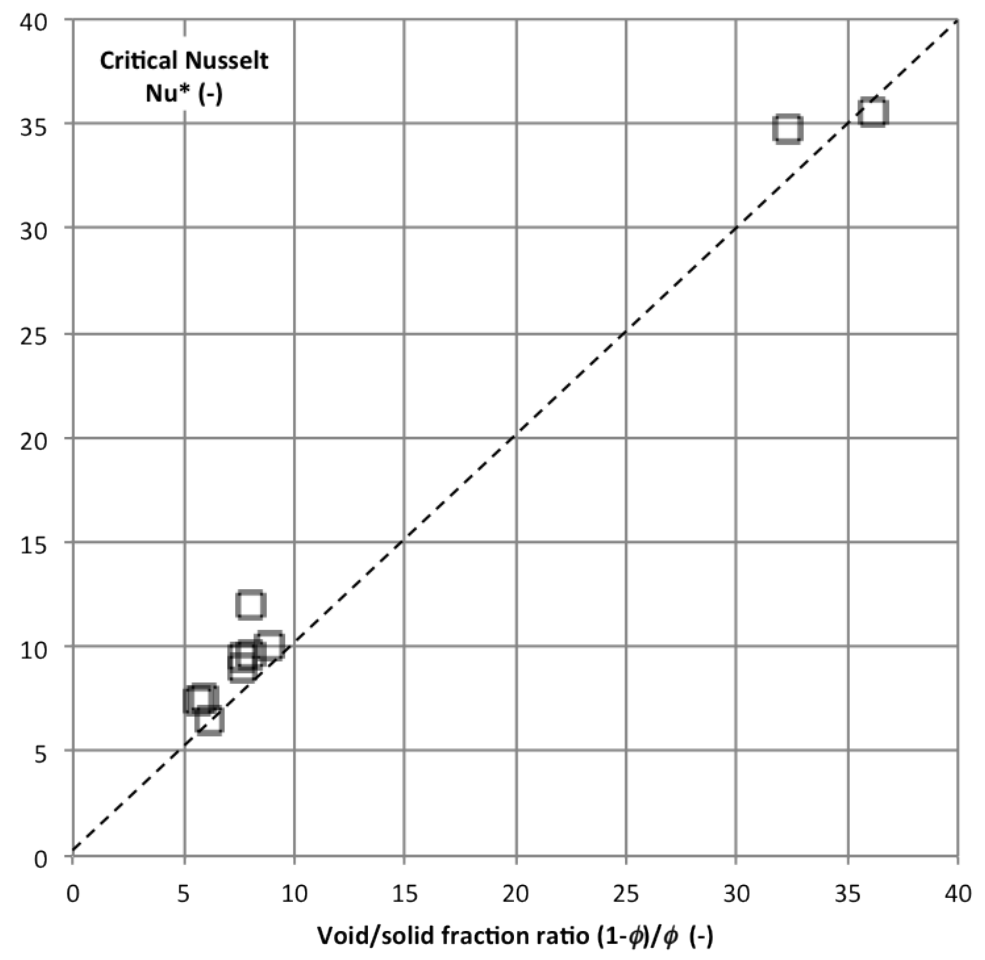

Figure 8: Critical Nusselt number vs. the void/solid fraction ratio for all studied foams. The dotted line represents equality.

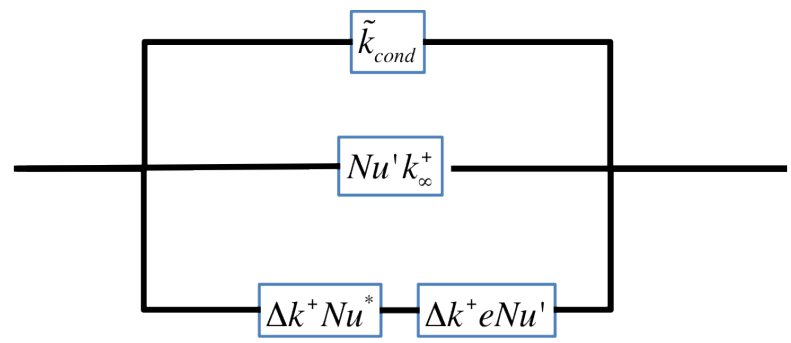

Figure 9: Equivalent circuit for heat transfer in mixed radiative/conductive mode. 
media is described using three tensorial parameters : $\tilde{k}_{\text {cond }} / \Phi, k_{\infty}^{+}$and $\Delta k^{+}$, and one scalar parameter $N u^{*}$, all appearing in equ. (4). This description is richer than the usual correlations given in literature because it is found here that $k^{+}$(or equivalently the scaled optical thickness) is a more complex function of emissivity and radiation/conduction ratio.

\subsection{Influence of structural parameters on the effective conductivity}

The values of $\tilde{k}_{\text {cond }} / \Phi, k_{\infty}^{+}, \Delta k^{+}$and $N u^{*}$ are summarized in the following tables. Table 3 summarizes all foams with solid phase amount $\Phi$ close to $15 \%$, Table 4 for all foams close to $11 \%$, Table 5 for the two foams with $3 \%$ solid.

\begin{tabular}{|c|c|c|c|c|}
\hline & & \multirow{2}{*}{$\begin{array}{l}\text { Actual foam } \\
\text { A_015 }\end{array}$} & \multicolumn{2}{|c|}{ Model foams (tetrakaidecahedra) } \\
\hline & & & IS O_015 & 557_015 \\
\hline \multirow[t]{2}{*}{$\begin{array}{l}\text { Pure } \\
\text { conduction } \\
\tilde{k}_{\text {cond }} / \Phi\end{array}$} & $\begin{array}{l}\text { Slowest }(\mathrm{X}) \\
\text { Intermediate } \\
\text { Fastest }(\mathrm{Z})\end{array}$ & $\begin{array}{l}0.43 \\
0.47 \\
0.48\end{array}$ & 0.51 & $\begin{array}{l}0.44 \\
0.60\end{array}$ \\
\hline & $\mathrm{Z} / \mathrm{X}$ ratio & 1.10 & 1 & 1.35 \\
\hline \multirow{3}{*}{$\begin{array}{l}\text { Pure } \\
\text { radiation } \\
\text { slope } k_{\infty}^{+}\end{array}$} & $\begin{array}{l}\text { Slowest }(\mathrm{X}) \\
\text { Intermediate }\end{array}$ & $\begin{array}{l}1.30 \\
1.38\end{array}$ & \multirow[t]{2}{*}{1.15} & 1.11 \\
\hline & Fastest (Z) & 1.43 & & 1.31 \\
\hline & $\mathrm{Z} / \mathrm{X}$ ratio & 1.10 & 1 & 1.18 \\
\hline \multirow{3}{*}{$\begin{array}{l}\text { Low } \\
\text { radiation } \\
\text { additional } \\
\text { slope } \Delta k^{+}\end{array}$} & $\begin{array}{l}\text { Slowest }(\mathrm{X}) \\
\text { Intermediate }\end{array}$ & $\begin{array}{l}1.35 \\
1.36\end{array}$ & \multirow[t]{2}{*}{1.06} & 1.03 \\
\hline & Fastest (Z) & 1.38 & & 1.06 \\
\hline & $\mathrm{Z} / \mathrm{X}$ ratio & 1.02 & 1 & 1.03 \\
\hline \multicolumn{2}{|c|}{ Critical ratio $N u^{*}$} & 6.6 & 7.5 & 7.6 \\
\hline
\end{tabular}

Table 3: Foams with $15 \%$ solid 


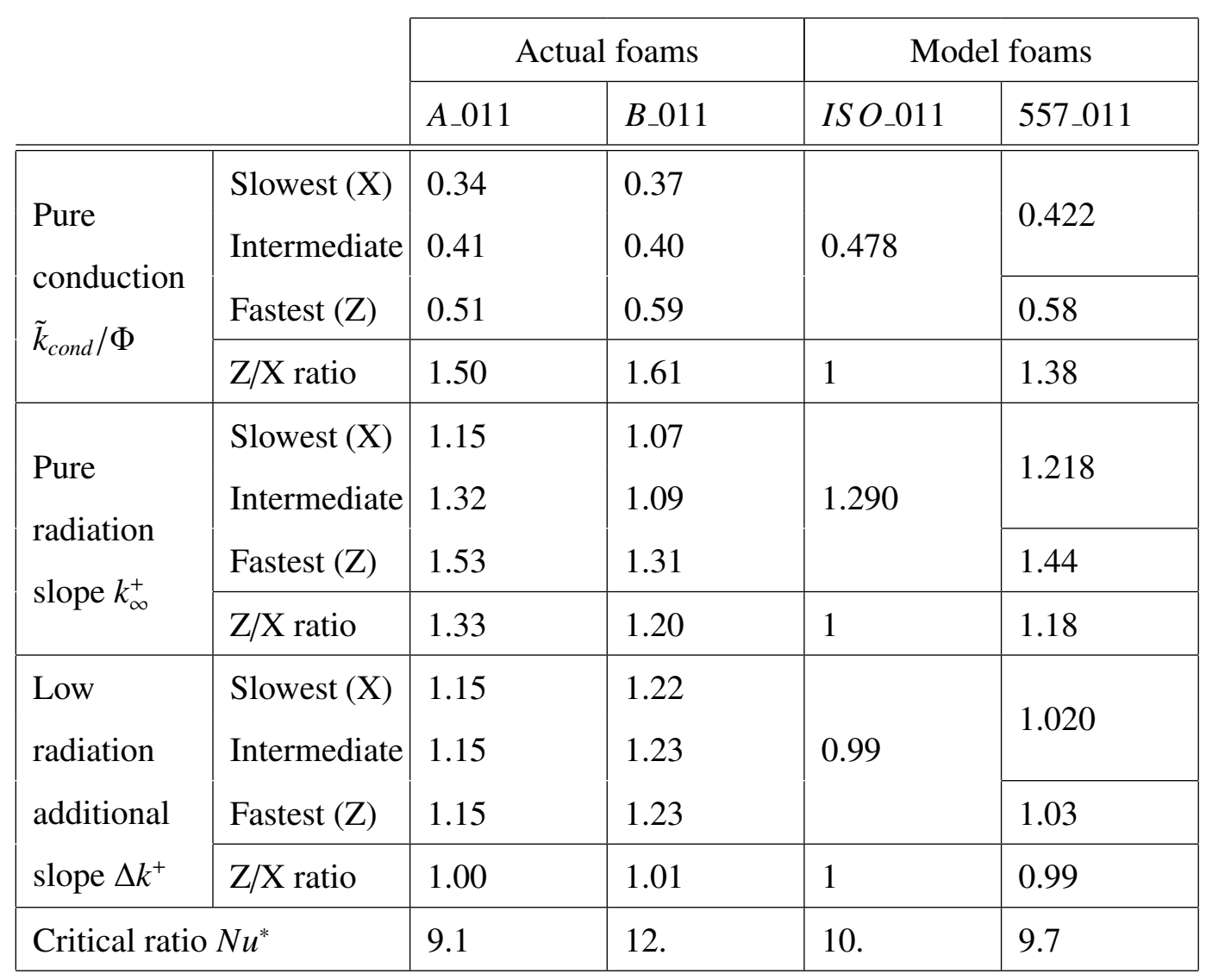

Table 4: Foams with $11 \%$ solid 


\begin{tabular}{|c|c|c|c|}
\hline & & \multicolumn{2}{|c|}{ Model foams (tetrakaidecahedra) } \\
\hline & & $\begin{array}{l}\text { 557_003 } \\
\text { open }\end{array}$ & $\begin{array}{l}557 \_003 C \\
\text { closed }\end{array}$ \\
\hline \multirow{3}{*}{$\begin{array}{l}\text { Pure conduction } \\
\tilde{k}_{\text {cond }} / \Phi\end{array}$} & Slowest (X) & 0.37 & 0.38 \\
\hline & Fastest (Z) & 0.55 & 0.51 \\
\hline & $\mathrm{Z} / \mathrm{X}$ ratio & 1.49 & 1.33 \\
\hline \multirow{3}{*}{$\begin{array}{l}\text { Pure radiation } \\
\text { slope } k_{\infty}^{+}\end{array}$} & Slowest (X) & 1.55 & 1.56 \\
\hline & Fastest (Z) & 1.81 & 1.61 \\
\hline & $\mathrm{Z} / \mathrm{X}$ ratio & 1.17 & 1.03 \\
\hline \multirow{3}{*}{$\begin{array}{l}\text { Low radiation } \\
\text { add. slope } \Delta k^{+}\end{array}$} & Slowest (X) & 0.93 & 0.91 \\
\hline & Fastest (Z) & 0.92 & 0.93 \\
\hline & $\mathrm{Z} / \mathrm{X}$ ratio & 1.01 & 1.02 \\
\hline \multicolumn{2}{|l|}{ Critical ratio $N u^{*}$} & 36. & 35. \\
\hline
\end{tabular}

Table 5: Foams with 3\% solid 
Some comments may be done about these results.

First, the scaled limit conductivities in purely conductive regime are linear in $\Phi$ (i.e. $\tilde{k} / \Phi$ is roughly constant) with a proportionality coefficient close to 0.35 0.5. The Lemlich theory [59] would predict $1 / 3$ for this value; Glicksman \& Schuetz [60] have predicted $(2-\varphi) / 3$ where $\varphi$ denotes a deviation from a purely reticulated character, in better agreement with our numerical findings.

Second, the fastest/slowest conductivity ratio for the "5:5:7" foams is almost exactly 1.4 , i.e. $7 / 5$, as expected, for the $15 \%$-solid foams. Then it increases towards 1.5 for the $3 \%$-solid, possibly due to a numerical discretization effect.

Third, all the pure radiation slopes $k_{\infty}^{+}$lie between 1.0 and 1.9, the largest values being for the 3\%-solid foams. They are sensitive to the anisotropy, but in a lesser extent than the purely conductive part - the $Z / X$ ratios are close to 1.2 . The anisotropy ratio is exactly the same for the three stretched tetrakaidecahedra.

Fourth, the low-radiation emissivity-dependent slope increment $\Delta k^{+}$is - rather surprisingly - isotropic. Moreover, though always close to 1, it has a clear dependence to the solid volume fraction. It seems to be larger in actual foams (between 1.15 and 1.35) than in ideal foams (between 0.91 and 1.06).

Finally, closing some windows in the 3\%-solid foam has the clear effect of $(i)$ making conduction more isotropic, and (ii) limiting the radiative contribution in the direction of the normal to the window shutting planes (i.e. the $Z$ direction), therefore making the medium more isotropic in the radiative regime too. No effect of the windows on $\Delta k^{+}$has been detected.

\section{Influence of the dominating heat transfer type on the effective conductivity eigendirections}

In ideal foams, the principal directions of heat transfer accompany, without surprise, the principal image directions (i.e. the $x, y$, and $z$ axes of the images); but 
on some actual media, their behavior is more complicated. Sample $A \_015$ contains some closed windows and displays an interesting variation of the eigendirection when going from the pure conduction mode to the radiation-dominated mode. Figure 10 illustrates this effect.

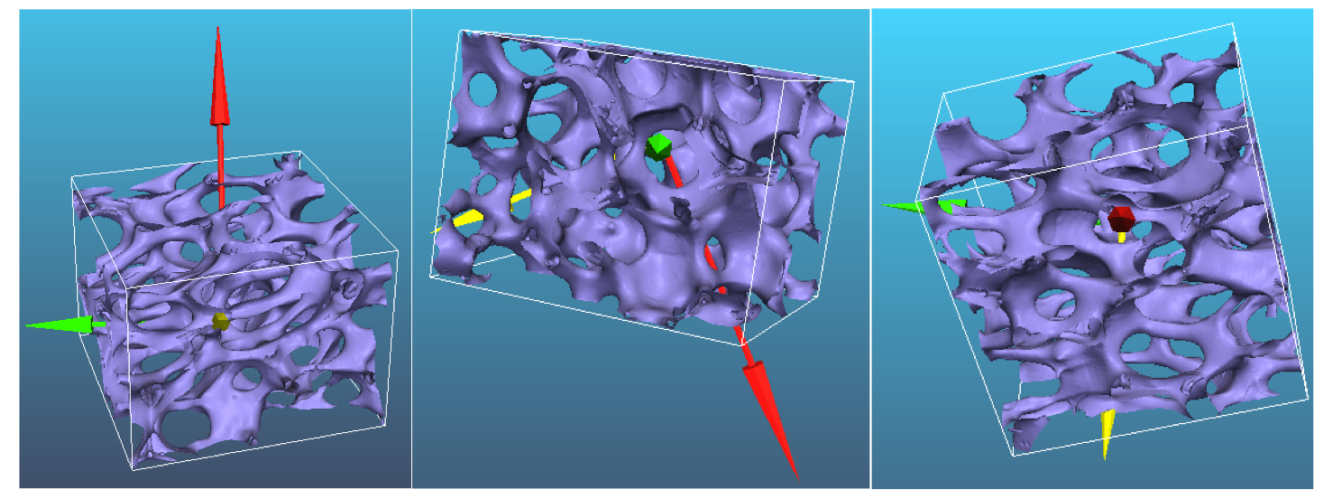

a)

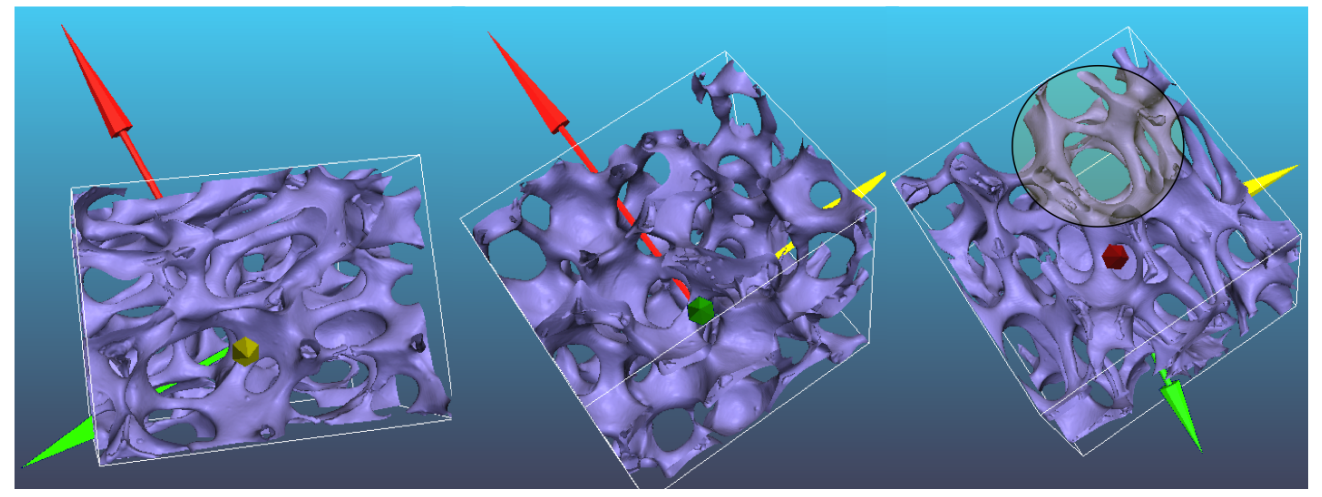

b)

Figure 10: Rendering of the principal directions of heat transfer in a) conduction-dominated and b) radiation-dominated modes. Red : fastest, Green: intermediate; Yellow : slowest direction.

The fastest eigendirection in conductive regime (see Figure 10 a)) seems to follow a path containing most window-closing walls. This was to be expected from the results of the preceding section obtained by comparing the $3 \%$-solid foams with open and partially closed windows. On the other hand, in the radiation- 
dominated mode (see Figure $10 \mathrm{~b}$ )), the eigendirections are strongly different. In this case, it is found out that the fastest direction coincides with the axis along which the widest windows are best matching each other. The circled pattern on the rightmost image of Figure $10 \mathrm{~b}$ ) highlights a "tunnel" through which radiative transfer is very efficient. In brief, this eigendirection analysis has allowed identification of some elements of the structure-property relationship. Highlighting this effect was only possible in sample $A_{-} \_15$ because it has window-closing walls and open window alignments that do not follow the symmetry of the principal axes.

\section{Conclusion}

This paper has presented the results of numerical computations of effective heat conductivity in 3D images of open-cell foams, considering the coupling of radiation and conduction thanks to a recent original simulation tool [53]. Actual and ideal (tetrakaidecahedral) foams were considered and compared. The radiative contribution to the effective conductivity is shown to vary with the radiation/conduction ratio, and a new analytical model has been fitted to the results. At very low radiation/conduction ratios, the conductivity is purely driven by conduction. At moderately low radiation/conduction ratios, there is an excess radiative conductivity, which is a function of the emissivity ; at high ratios, the radiative conductivity does not depend any more on the emissivity. The critical radiation/conduction ratio separating the latter two regimes matches the cell/strut diameter ratio. This is the first time that this variation has been evidenced, as the computational tool is also the first one to provide a strong, local, pore-scale coupling between radiation and conduction in porous media. Apparently, this behavior is expected to be more manifest in the case of extremely porous foams, since the critical radiation/conduction ratio would show up at higher temperatures. The influence of some structural features of the foams, namely the existence of some 
window-closing walls and of open channels, has been captured not only through the values of the ETC in the principal directions but also through the evolution of the eigendirections when the conduction/radiation ratio changes. Future work includes the comparison to actual experimental data, which necessitates a careful identification of the emissivity of the solid phase on the one hand, and of the ETC over a broad temperature domain. Also, extension of the software capability to the case of fluid-filled foams is of prime interest; indeed, in many practical cases, the gas or liquid saturating the pore space contributes significantly to the overall ETC.

\section{Nomenclature}

\begin{tabular}{|l|l|l|}
\hline Symbol & Meaning & Unit \\
\hline$d$ & Diameter & $\mathrm{m}$ \\
$k$ & Thermal conductivity & $\mathrm{W} \cdot \mathrm{m}^{-1} \cdot \mathrm{K}^{-1}$ \\
$\hat{k}$ & Scaled thermal conductivity & - \\
$L_{x, y \text { or } z}$ & Cell dimensions & $\mathrm{m}$ \\
$L_{p i x}$ & Pixel size & $\mathrm{m}$ \\
$N_{x, y \text { or } z}$ & Cell dimensions & voxels \\
$N u^{\prime}$ & Nusselt number & - \\
$N u^{*}$ & Critical Nusselt number & - \\
$S_{v}$ & Surface area per unit volume & $\mathrm{m}^{-1}$ \\
$T$ & Temperature & $\mathrm{K}$ \\
\hline
\end{tabular}




\begin{tabular}{|l|l|l|}
\hline Symbol & Meaning & Unit \\
\hline$\Delta k^{+}$ & Scaled radiative conductivity slope excess & - \\
$\varepsilon$ & Wall emissivity & - \\
$\Pi$ & Porosity & - \\
$\rho$ & Density & $\mathrm{kg} \cdot \mathrm{m}^{-3}$ \\
$\sigma$ & Stefan's constant & $\mathrm{W} \cdot \mathrm{m}^{-2} \cdot \mathrm{K}^{-4}$ \\
$\Phi$ & Relative density & - \\
\hline
\end{tabular}

\begin{tabular}{|l|l|l|}
\hline Symbol & Meaning & Unit \\
\hline$\bullet_{c}$ & relative to cells & \\
$\bullet_{\text {eff }}$ & effective & \\
$\bullet_{\text {ref }}$ & reference & \\
$\bullet_{\text {rad }}$ & radiative & \\
$\bullet_{s}$ & relative to the solid phase & \\
$\bullet_{\text {st }}$ & relative to struts & \\
$\bullet^{+}$ & radiation-related & \\
$\bullet_{\infty}$ & in limiting regime & \\
\hline
\end{tabular}




\section{References}

[1] L. Gibson, M. Ashby, Cellular Solids: Structure and Properties, 2nd Edition, University Press, Cambridge, United Kingdom, 1997.

[2] E. Placido, M. Arduini-Schuster, J. Kuhn, Thermal properties predictive model for insulating foams, Infrared Phys. Tech. 46 (2005) 219-231.

[3] R. Coquard, D. Baillis, Modeling of heat transfer in low-density eps foams, Int. J. Heat Mass Transfer 128 (2006) 538-549.

[4] A. Kaemmerlen, C. Vo, F. Asllanaj, G. Jeandel, D. Baillis, Radiative properties of extruded polystyrene foams: predictive model and experimental result, J. Quant. Spectrosc. Rad. Transf. 111 (2010) 865-877.

[5] K. Boomsma, D. Poulikakos, F. Zwick, Metal foams as compact high performance heat exchangers, Mech. Mater. 35 (2003) 1161-1176.

[6] M. Kaplan, M. Hall, The combustion of liquid fuels within a porous media radiant burner, Exp. Therm. Fluid Sci. 11 (1995) 13-20.

[7] J. Randrianalisoa, Y. Bréchet, D. Baillis, Materials selection for optimal design of a porous radiant burner for environmentally driven requirements, Adv. Eng. Mater. 11 (2009) 1049-1056.

[8] A. Steinfeld, R. Palumbo, Solar thermochemical process technology, Vol. 15 of Encyclopedia of physical science and technology, Academic Press, New York, 2001, pp. 237-256.

[9] T. Fend, B. Hoffschmidt, R. Pitz-Paal, O. Reutter, P. Rietbrock, Porous materials as open volumetric solar receivers: experimental determination of thermo- physical and heat transfer properties, Energy 29 (2004) 823-833. 
[10] P. Furler, J. Scheffe, M. Gorbar, M. Moes, U. Vogt, A. Steinfeld, Solar thermochemical $\mathrm{CO}_{2}$ splitting utilizing a reticulated porous ceria redox system, Energy \& Fuels 26 (2012) 7051-7059.

[11] Q. Wang, X.-H. Han, A. Sommers, Y. Park, C. Joen, A. Jacobi, A review on application of carbonaceous materials and carbon matrix composites for heat exchangers and heat sinks, Int. J. Refrig. 35 (2012) 7-26.

[12] V. Norvell, G. Mamantov, Optically transparent vitreous carbon electrode, Anal. Chem. 49 (1977) 1470-1472.

[13] J. Wang, Reticulated vitreous carbon-a new versatile electrode material, Electrochimica Acta 26 (1981) 1721-1726.

[14] M. Inagaki, F. Kang, Materials Science and Engineering of Carbon, Elsevier, 2014.

[15] S.-T. Hong, D. Herling, Open-cell aluminum foams filled with phase change materials as compact heat sinks, Scripta Mater. 55 (2006) 887-890.

[16] A. Fedorov, R. Viskanta, Radiative transfer in a semitransparent glass foam blanket, Phys. Chem. Glasses 41 (3) (2000) 127-135.

[17] R. Coquard, D. Baillis, Radiative and conductive thermal properties of foams, in: A. Öchsner, G. E. Murch, M. de Lemos (Eds.), Thermal Properties of Cellular and Porous Materials, Wiley-VCH, Weinheim, 2008, pp. 343-384.

[18] P. Ranut, On the effective thermal conductivity of aluminum metal foams: Review and improvement of the available empirical and analytical models, Experimental Thermal and Fluid Science in press. DOI:10.1016/j.applthermaleng.2015.09.094. 
[19] R. Siegel, J. R. Howell, Thermal Radiation Heat Transfer, Taylor \& Francis, New York, 2002, Ch. 15.

[20] M. Arduini-Schuster, J. Manara, C. Vo, Experimental characterization and theoretical modeling of the infrared-optical properties and the thermal conductivity of foams, Int. J. Thermal Sci. 98 (2015) 156-164.

[21] M. A. Mendes, V. Skibina, P. Talukdar, R. Wulf, U. Gross, D. Trimis, S. Ray, Experimental validation of simplified conduction-radiation models for evaluation of effective thermal conductivity of open-cell metal foams at high temperatures, Int. J. Heat \& Mass Transfer 78 (2014) 112-120.

[22] C. D. Micco, C. Aldao, Radiation contribution to the thermal conductivity of plastic foams, J. Polymer Sci B : Polymer Phys. 43 (2005) 190-192.

[23] O. Almanza, M. R. Pérez, J. D. Saja, Prediction of the radiation term in the thermal conductivity of crosslinked closed cell polyolefin foams, J. Polymer Sci B : Polymer Phys. 38 (2000) 993-1004.

[24] M. A. Mendes, S. Ray, D. Trimis, An improved model for the effective thermal conductivity of open-cell porous foams, Int. J. Heat \& Mass Transfer 75 (2014) 224-230.

[25] S. Delettrez, Elaboration par voie gazeuse et caractérisation de céramiques alvéolaires base pyrocarbone ou carbure de silicium, Ph.D. thesis, University Bordeaux I (2008).

[26] H. W. Russell, Principles of heat flow in porous insulators, J. Amer. Ceram. Soc. 18 (1935) 1-5.

[27] X. Fu, R. Viskanta, J. Gore, Prediction of effective thermal conductivity of 
cellular ceramics, Int. Commun. Heat \& Mass Transfer 25 (2) (1998) 151160.

[28] V. V. Calmidi, R. L. Mahajan, The effective thermal conductivity of high porosity fibrous metal foams, J. Heat Transfer 121 (2) (1999) 466-471.

[29] A. Bhattacharya, V. Calmidi, R. Mahajan, Thermophysical properties of high porosity metal foams, Int. J. Heat \& Mass Transfer 45 (5) (2002) 10171031.

[30] R. Singh, H. Kasana, Computational aspects of effective thermal conductivity of highly porous metal foams, Appl. Thermal Eng. 24 (13) (2004) 1841 -1849 .

[31] J.-F. Wang, J. Carson, J. Willix, M. North, D. Cleland, A symmetric and interconnected skeleton structural (SISS) model for predicting thermal and electrical conductivity and young's modulus of porous foams, Acta Mater. 56 (2008) 5138-5146.

[32] K. Boomsma, D. Poulikakos, On the effective thermal conductivity of a three-dimensionally structured fluid-saturated metal foam, Int. J. Heat \& Mass Transfer 44 (2001) 827-836.

[33] Z. Dai, K. Nawaz, Y. Park, J. Bock, A. Jacobi, Correcting and extending the Boomsma-Poulikakos effective thermal conductivity model for threedimensional, fluid-saturated metal foams, Int. Commun. Heat \& Mass Transfer 37 (6) (2010) 575-580.

[34] K. Boomsma, D. Poulikakos, Corrigendum to "On the effective thermal conductivity of a three-dimensionally structured fluid-saturated metal foam", Int. J. Heat \& Mass Transfer 54 (2011) 746-748. 
[35] Y.-P. Yao, H.-Y. Wu, Z.-Y. Liu, A new prediction model for the effective thermal conductivity of high porosity open-cell metal foams, Int. J. Thermal Sci. 97 (2015) 56-67.

[36] P. Kumar, F. Topin, Simultaneous determination of intrinsic solid phase conductivity and effective thermal conductivity of kelvin like foams, Appl. Thermal Eng. 71 (2014) 536-547.

[37] J. Randrianalisoa, D. Baillis, C. L. Martin, R. Dendievel, Microstructure effects on thermal conductivity of open-cell foams generated from the Laguerre-Voronoï tessellation method, Int. J. Thermal Sci. 78 (2015) 277286.

[38] K. K. Bodla, J. Y. Murthy, S. V. Garimella, Resistance network-based thermal conductivity model for metal foams, Comput. Mater. Sci. 50 (2010) $622-632$.

[39] R. Coquard, D. Baillis, Numerical investigation of conductive heat transfer in high-porosity foams, Acta Mater. 57 (18) (2009) 5466-5479.

[40] P. Ranut, E. Nobile, L. Mancini, High resolution X-ray microtomographybased CFD simulation for the characterization of flow permeability and effective thermal conductivity of aluminum metal foams, Exp. Thermal \& Fluid Sci. 67 (2015) 30—36.

[41] M. Loretz, R. Coquard, D. Baillis, E. Maire, Metallic foams : Radiative properties/comparison between different models, J. Quant. Spectrosc. \& Rad. Transf. 109 (2008) 16-27.

[42] J. Petrasch, P. Wyss, A. Steinfeld, Tomography-based monte carlo determination of radiative properties of reticulate porous ceramics, J. Quant. Spectrosc. \& Rad. Transf. 105 (2) (2007) 180—197. 
[43] B. Rousseau, D. de Sousa Meneses, P. Echegut, J. Thovert, Textural parameters influencing the radiative properties of a semitransparent porous media, Int. J. Thermal Sci. 50 (2) (2011) 178-186.

[44] R. Coquard, B. Rousseau, P. Echegut, D. Baillis, H. Gomart, E. Iacona, Investigations of the radiative properties of al-nip foams tomographic images and stereoscopic micrographs, Int. J. Heat \& Mass Transfer 55 (2012) $1606-1619$.

[45] M. Tancrez, J. Taine, Direct identification of absorption and scattering coefficients and phase function of a porous medium by a Monte Carlo technique, Int. J. Heat \& Mass Transfer 47 (2) (2004) 373-383.

[46] J. R. Howell, M. Perlmutter, The calculation of nonlinear radiation transport by a Monte Carlo method: Statistical physics, Meth. in Comput. Phys. 1 (1961) 43-65.

[47] S. Cunsolo, R. Coquard, D. Baillis, N. Bianco, Radiative properties modeling of open cell solid foam: review and new analytical law, Int. J. Thermal Sci. 104 (2016) 122-134.

[48] L. Trovalet, G. Jeandel, P. Coelho, F. Asllanaj, Modified finite-volume method based on a cell vertex scheme for the solution of radiative transfer problems in complex 3D geometries, J. Quant. Spectrosc. \& Rad. Transfer 112 (17) (2011) 2661-2675.

[49] R. Coquard, D. Rochais, D. Baillis, Conductive and radiative heat transfer in ceramic and metal foams at fire temperatures, Fire Technol. 48 (2012) $699-732$.

[50] M. Chahlafi, F. Bellet, F. Fichot, J. Taine, Radiative transfer within non Beerian porous media with semitransparent and opaque phases in non equilib- 
rium: Application to reflooding of a nuclear reactor., Int. J. Heat \& Mass Transfer 55 (13-14) (2012) 3666-3676.

[51] M. Zarrouati, F. Enguehard, J. Taine, Radiative transfer within strongly non homogeneous porous media: Application to a slab of packed particles, Int. J. Heat \& Mass Transfer 91 (2015) 936-947.

[52] V. Leroy, B. Goyeau, J. Taine, Coupled upscaling approaches for conduction, convection, and radiation in porous media: Theoretical developments, Transp. in Porous Med. 98 (2) (2013) 323-347.

[53] G. L. Vignoles, A hybrid random walk method for the simulation of coupled conduction and linearized radiation transfer at local scale in porous media with opaque solid phases, Int. J. Heat \& Mass Transfer 93 (2016) 707-719.

[54] A. Ortona, S. Pusterla, P. Fino, F. R. A. Mach, A. Delgado, S. Biamino, Aging of reticulated $S i-S i C$ foams in porous burners, Adv. Appl. Ceram. 109 (4) (2010) 246-251.

[55] C. D’Angelo, A. Ortona, Cellular Ceramics Produced by Replication:A Digital Approach, Adv. Eng. Mater. 14 (12) (2012) 1104-1109.

[56] G. L. Vignoles, M. Donias, C. Mulat, C. Germain, J.-F. Delesse, Simplified marching cubes: An efficient discretization scheme for simulations of deposition/ablation in complex media, Computational Materials Science 50 (3) (2011) 893 - 902.

[57] M. F. Modest, Radiative Heat Transfer, $3^{\text {rd }}$ Edition, Academic Press, New York, S. Francisco, 2013.

[58] J. Taine, F. Bellet, V. Leroy, E. Iacona, Generalized radiative transfer equa- 
tion for porous medium upscaling: Application to the radiative Fourier laws, Int. J. Heat \& Mass Transfer 53 (19-20) (2010) 4071-4081.

[59] R. Lemlich,A theory for the limiting conductivity of polyhedral foam at low density, J. Colloid \& Interface Sci. 64 (1) (1978) 107 - 110.

[60] L. Glicksman, M. A. Schuetz, Heat Transfer in foams, in "Low Density Cellular Plastics, N.C. Hilyard and A. Cunningham eds., Springer Netherlands, Dordrecht, 1994, pp. 105-152. 